\title{
Microleakage Evaluation in Class V Cavities Restored with Five Different Resin Composites: In vitro Dye Leakage Study
}

\author{
Sahar Bajabaa' \\ Shaza Balbaid' \\ Muruj Taleb' \\ Lujain Islam' \\ Salem Elharazeen ${ }^{2}$ \\ Ebaa Alagha (ID)
}

'Restorative Department, Vision Colleges, Jeddah, Saudi Arabia; ${ }^{2}$ Shiyar

Medical Complex, Jeddah, Saudi Arabia
Correspondence: Ebaa Alagha

Email drebaaialagha@gmail.com
Purpose: Restorative materials and techniques have improved the clinical success of a variety of restorative procedures. Despite these new improvements, microleakage remains one of the leading causes of restoration failure and may lead to postoperative sensitivity, enamel microcracks, marginal staining, discoloration, recurrent caries, and deformation of teeth. This study evaluated microleakages of five recent resin composites in class $\mathrm{V}$ cavities. Materials and Methods: Standardized class V cavities were prepared on the labial surfaces of one hundred extracted intact and noncarious human permanent premolar teeth. The cavities were divided into five groups $(n=20)$ according to the resin composite material. Groups: A1, A2, A3, A4 and A5 were restored with Omnichroma, Spectrum, Mosaic, Tetric $\mathrm{N}$-Ceram and Harmonize, respectively. The teeth were stained with methylene blue and then sectioned, and the extent of dye penetration was examined under a stereomicroscope and scanning electron microscope (SEM) to evaluate microleakage. One-way ANOVA test was used to statistically analyze the data. Statistical Package for Social Sciences Computer Software (SPSS) version 15.0 was used.

Results: Dye penetration was observed at the tooth-restoration interface in all the studied samples, with varying degrees of penetration. At the cervical margins of the cavities, no statistically significant difference in the microleakage scores was observed $(\mathrm{P}=0.16)$. At the cavity floor, no statistically significant difference in microleakage scores was observed $(\mathrm{P}=0.74)$. Omnichroma resin composite had the highest microleakage results.

Conclusion: Nanohybrid resin composites showed less microleakage than other resin composites. Among all the groups, Omnichroma showed the highest microleakage at the cavity floor and at the cervical areas.

Clinical Significance: In light of our findings, nanohybrid resin composites appear to be capable of reducing microleakage, and the results reported herein must be verified by additional clinical trials.

Keywords: class V, microleakage, resin composite, scanning electron microscope

\section{Introduction}

Composite resins are promising tooth-resembling materials in restorative dentistry. ${ }^{1}$ Composite resins remain the preferred choice of many dentists due to their positive characteristics, such as aesthetics, adhesion, and tooth structure preservation. However, resin composites have several limitations, including method sensitivity, polymerization shrinkage, and the possibility of microleakage and secondary caries. $^{2}$ Recently, new materials with enhanced formulations, characteristics and 
appearance have made the placement of direct composites more reliable and predictable. ${ }^{1}$ Restoring carious or noncarious lesions in the cervical area is still considered challenging in the dental clinics. One of the difficulties associated with class $\mathrm{V}$ cavities is tooth isolation, due to morphological characteristics of the cervical region which limits placement of the rubber dam and clamp. ${ }^{3}$ Another difficulty in such cavities is that if the restorative material does not adhere to dentin or cementum, microleakages result that allow bacteria to pass through this gap between the cavity wall and resin composite and may lead to hypersensitivity, secondary caries, pulpal pathosis and eventually failure of restorations. ${ }^{4}$ Microleakage is the main factor that affects dental restoration longevity. ${ }^{5}$ Modern adhesive dentistry aims to reduce microleakage by enhancing the marginal adaptation of dental restoration. ${ }^{6}$

To the best of our knowledge, no study has evaluated the microleakage of Omnichroma resin composites; therefore, this work aimed to evaluate the microleakage of five recent resin composites in class $\mathrm{V}$ cavities. The null hypothesis of this study was that there would not be a difference in the levels of microleakage between different types of resin composites.

\section{Materials and Methods}

Sample size determination was performed using G Power v3.1.3 software (University of Düsseldorf; Düsseldorf, Germany). A power analysis revealed that a sample size of 20 per group was found to meet the constraints of $\alpha=0.05$ and power $=0.80$. One hundred noncarious, intact human permanent upper premolar teeth were collected. The teeth were scaled and cleaned with tap water for surface debridement, polished with a rubber cup and pumice, and stored in distilled water at room temperature until they were used in the study. The study was conducted in full accordance with the World Medical Association Declaration of Helsinki with the approval of the Ethics Committee of Vision College (approval number: 20-3/8). Informed consent of the patient or the patient's authorized representative prior to the extraction procedure was obtained from the oral surgery departmentvision colleges. According to the type of resin composite, the teeth were randomly divided into five groups of 20 teeth each $(n=20)$. On the buccal surface of each tooth, Class V cavities were performed in the gingival one-third. The cervical margin was located $0.5 \mathrm{~mm}$ apical to the cementoenamel junction (on dentin/cementum). The cavities in the dentin were $2 \mathrm{~mm}$ in diameter and $2 \mathrm{~mm}$ in depth. Under a water coolant, a round diamond bur size BR-45, ISO-001/010 (Beijing, China) was utilized with a high-speed hand piece (NSK Pana Max, Japan) to standardize the cavity width. By inserting the full head of the bur, the cavity's depth was adjusted to $2 \mathrm{~mm}$. To control the depth, an endodontic file stopper was placed at the end of the bur head. At any of the prepared cavity enamel margins, no bevels were produced. ${ }^{7}$ To eliminate dullness, a new bur was used for each cavity. ${ }^{8}$ One component selfetching light cure adhesive (Bond Force, Tokuyama Dental) was applied with a fully saturated microbrush with slight agitation to cover the entire surface and was gently air dried approximately $0.5 \mathrm{~mm}$ away from the prepared surface for 1-3 seconds, to allow the solvent to evaporate. The adhesive was then cured using a light curing unit (LED.B which has high-power LED blue light, rechargeable battery with AC $100 \mathrm{~V}-240 \mathrm{~V} 50 \mathrm{~Hz} /$ $60 \mathrm{~Hz}$ and wavelength range of: $420 \mathrm{~nm}-480 \mathrm{~nm}$ ) for 20 seconds according to the manufacturer's instructions. ${ }^{9}$ Group A1 was restored with Omnichroma (Tokuyama, Japan), Group A2 was restored with Spectrum (Dentsply, United States), Group A3 was restored with Mosaic (Ultradent, Germany), Group A4 was restored with Tetric N-Ceram (Ivoclar Vivadent, Liechtenstein) and Group A5 was restored with Harmonize (Kerr, United States). A2 color shade was used for all types of composite resin except for Omnichroma which has only one shade in the dental market. Data on the resin composite materials are shown in Table 1. To avoid composite sticking to the instrument, the restorations were placed in two increments using a plastic condenser (Composite placement instrument, 636, 3 M ESPE dental products, USA) and cured for 20 seconds according to the manufacturer's instructions. The teeth underwent thermocycling for 1000 cycles in a water bath at $5^{\circ}$ and $55^{\circ} \mathrm{C}$ for 30 seconds. ${ }^{10}$ The entire tooth surface was covered with two layers of nail varnish (essence shine last and go, gel nail polish) within $1 \mathrm{~mm}$ of the bonded interface and left undisturbed for one day to allow the varnish to dry. ${ }^{11}$ The apices of the roots were sealed using sticky modeling wax (Cavex, Holland). Each tooth was then wrapped with aluminum foil, which was adapted using a ball burnisher. A window in the aluminum foil was cut so that the restoration and $1 \mathrm{~mm}$ around it was exposed using a sharp scalpel. A final coat of nail varnish was applied on the wrapped foil at the cut edges to ensure proper sealing. Teeth were immersed in a freshly prepared 
Table I Resin Composite Materials Used in the Study

\begin{tabular}{|c|c|c|c|c|}
\hline Material & Symbol & Product & Composition* & Company \\
\hline \multirow[t]{5}{*}{$\begin{array}{l}\text { Types of Resin } \\
\text { composites }\end{array}$} & Al & Omnichroma & $\begin{array}{l}\text { Universal supra-nano spherical filled resin composite contains: } \\
\text {-Uniform sized supra-nano spherical filler ( } 260 \mathrm{~nm} \text { spherical SiO2-ZrO2) } \\
\text {-UDMA, TEGDMA } \\
\text {-Filler load: } 79 \text { wt\% (68 vol\%) }\end{array}$ & $\begin{array}{c}\text { Tokuyama } \\
\text { Dental }\end{array}$ \\
\hline & $\mathrm{A} 2$ & Spectrum & $\begin{array}{l}\text { Submicron hybrid composite contains: } \\
\text {-Glass, and/or fused silica or mixed oxides such as silica-zirconia } \\
\text {-Urethane modified Bis-GMA resin; TEGDMA; Dimethacrylate Resin } \\
\text {-Filler load: } 77.2 \mathrm{wt} \% / 57.0 \text { vol\% }\end{array}$ & Dentsply \\
\hline & A3 & Mosaic & $\begin{array}{l}\text { Nano-hybrid composite contains: } \\
\text {-Zirconia-silica glass, glass ceramic and } 20 \text { nanometer silica } \\
\text {-Bis-GMA } \\
\text {-Filler load: } 68 \text { vol\% }\end{array}$ & Ultradent \\
\hline & A4 & $\begin{array}{c}\text { Tetric } \\
\text { N-Ceram }\end{array}$ & $\begin{array}{l}\text { Nano-hybrid composite contains: } \\
\text {-Barium glass, ytterbium trifluoride, mixed oxide and copolymers -the particle size } \\
\text { is between ( } 40 \mathrm{~nm} \text { and } 3000 \mathrm{~nm}) \\
\text {-Dimethacrylates } \\
\text {-Filler load: } 80-81 \mathrm{wt} \%\end{array}$ & $\begin{array}{l}\text { Ivoclar } \\
\text { Vivadent }\end{array}$ \\
\hline & A5 & Harmonize & $\begin{array}{l}\text { Nano-hybrid universal composite contains: } \\
\text {-Silica; Zirconia; Barium Glass (400 nm) } \\
\text {-Bis-GMA; Bis-EMA; TEGDMA } \\
\text {-Filler load: } 8 \text { I wt } \%\end{array}$ & Kerr \\
\hline
\end{tabular}

Note: *According to information from the manufacturers.

aqueous methylene blue solution with a concentration of $2 \mathrm{gm} / 200$ c.c water for 4 hours at room temperature. ${ }^{12}$ The teeth were then removed from the dye and rinsed completely and gently under running water for three minutes to remove any remaining color. The aluminum foil wrapper was removed, and any leftovers on the teeth were thoroughly cleansed. The teeth were vertically sectioned through the center of the restoration, by a cutting machine (cutting machine, E96, USA) using a diamond disk (diamond disk, EDENTA Golden S.A.W, Swiss made) in a buccolingual direction along their long axis to assess the microleakage at the cervical margins. The sections were then separated, and the tooth restoration interface was examined at the cervical margins under a stereomicroscope (Nikon Eclipse E600, Tokyo, Japan), at $45 \mathrm{X}$ magnification interface ${ }^{13}$ in which the image of the restoration was captured and transferred to a computer equipped with the image analysis software program (ImageJ 1.43U, National Institute of Health, USA), where the leakage was evaluated as follows: ${ }^{14}$ Penetration $(\%)=($ traced dye/cavity $) * 100$.

The following scoring criteria have been used:. ${ }^{15}$

\section{$0=$ No microleakage}

$1=$ Microleakage at the $1 / 3 \mathrm{rd}$ initial side of the cavity wall

$2=$ Microleakage extending beyond the amelodentinal junction

$3=$ Microleakage along the floor of the cavity

4=Microleakage reaching the pulp.

After the microleakage test, 5 specimens were selected from each group and the tooth hard tissue and restorative material interface were examined under scanning electron microscope (SEM) (Seron, Aura100, Scanning Electron Microscope, Tokyo, Japan) and photographed.

\section{Results}

The mean microleakage in the cervical area for Omnichroma was the highest $(1.15 \pm 0.83)$ followed by Harmonize ( $0.75 \pm 0.13)$, Spectrum $(0.67 \pm 0.22)$, Mosaic $(0.50 \pm 0.25)$ and the least microleakage was seen for Tetric $\mathrm{N}$-Ceram $(0.42 \pm 0.26)$. Even though Tetric N-Ceram showed the least microleakage in the cervical area, no statistically significant difference was observed between the different materials $(\mathrm{P}=0.16)$. The mean microleakage 
at the cavity floor for Omnichroma had the highest microleakage $(2.18 \pm 0.25)$ followed by Spectrum $(2.05 \pm 0.13)$, Tetric N-Ceram (2.02 \pm 0.27$)$, Mosaic $(1.97 \pm 0.31)$ and Harmonize $(1.94 \pm 0.30)$. No statistically significant difference in microleakage was observed at the cavity floor between all materials. ( $\mathrm{P}=0.74)$ Table 2 .

The stereomicroscope photographs revealed that in all specimens, microleakage was manifested by methylene blue dye penetration to different degrees (Figure 1A-E). The findings were also supported by scanning electron microscopy (SEM) examination. Scanning electron photomicrographs at magnification of $100 \mathrm{X}$ are shown in Figure 1F-J.

\section{Discussion}

In vitro procedures should be used to test the clinical performance of any new restorative material. These procedures are used to assess leakage rather than in vivo approaches, which can be qualitative and quantitative. ${ }^{16}$ Many innovations in restorative materials have begun to appear on the dentistry market to improve the success rates of such materials. ${ }^{17}$ Nanotechnology is defined as the use of numerous physical and chemical approaches to create materials and structures in the range of $0.1-100 \mathrm{~nm} .{ }^{18}$ Based on the findings of the present study, the null hypothesis was accepted given that no significant difference in microleakage was noted between the different types of composite resins.

In this study, five recent resin composites were used because they are routinely used in dental practice, such as nanohybrid composites that contain $0.005-0.01 \mathrm{~nm}$ particles. ${ }^{19}$ These composite resins provide good aesthetic as well as satisfactory physical, chemical, and biological properties which are deemed necessary for an increased longevity of

Table 2 Comparison of Microleakage Between Different Restorative Materials at the Cervical Area and at the Cavity Floor

\begin{tabular}{|l|l|l|l|l|}
\hline \multirow{2}{*}{ Variables } & \multicolumn{2}{|c|}{ Cervical Area } & \multicolumn{2}{c|}{ Cavity Floor } \\
\cline { 2 - 5 } & Mean & \pm SD & Mean & \pm SD \\
\hline G I: Omnichroma & 1.15 & \pm 0.83 & 2.18 & \pm 0.25 \\
G 2: Spectrum & 0.67 & \pm 0.22 & 2.05 & \pm 0.13 \\
G 3: Mosaic & 0.50 & \pm 0.25 & 1.97 & \pm 0.31 \\
G 4: Tetric N-Ceram & 0.42 & \pm 0.26 & 2.02 & \pm 0.27 \\
G 5: Harmonize & 0.75 & \pm 0.13 & 1.94 & \pm 0.30 \\
\hline P-value & \multicolumn{3}{|c|}{$P=0.16$} & \multicolumn{2}{|c|}{0.74} \\
\hline
\end{tabular}
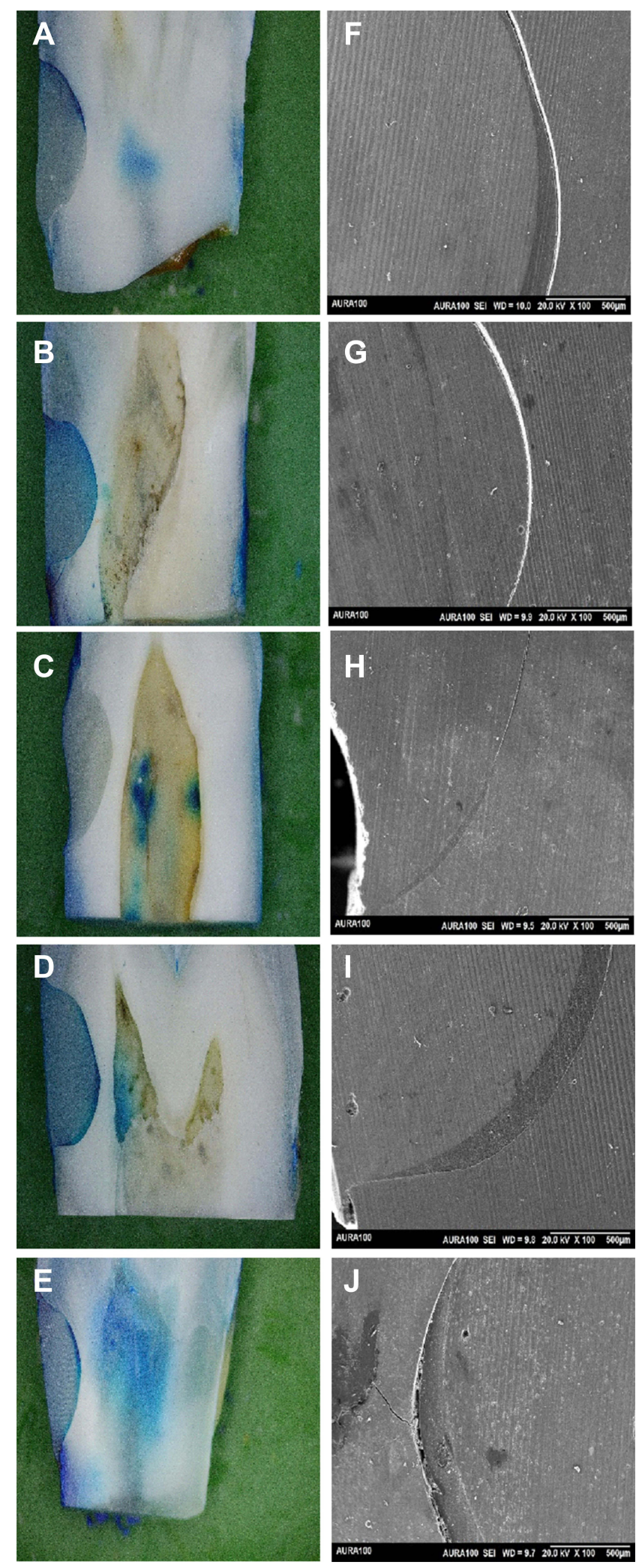

Figure I Stereomicroscope photographs of microleakage in (A) Omnichroma, (B) Spectrum, (C) Mosaic, (D) Tetric N-Ceram and (E) Harmonize. Scanning electron photomicrographs of microleakage in (F) Omnichroma, (G) Spectrum, $(\mathbf{H})$ Mosaic, (I) Tetric N-Ceram and (J) Harmonize. 
the composite restoration. The discrepancy in resin composite formulas was hypothesized to result in varying polymerization shrinkage ${ }^{5}$ which is the most common cause of failure in direct composite resin restorations. The amount of material in the polymerization reaction, the boundary conditions, and the formulation of the material all play a role in restoration failure. ${ }^{5}$ Restoration failure depends on cavity size and shape, substrate type and margin position, restorative material and placement and polymerization procedure. ${ }^{20}$ In this study, intact noncarious class $\mathrm{V}$ cavities were prepared as they are appropriate for microleakage evaluation given that they have a low configuration factor, and are easy to prepare and restore, reducing technique sensitivity. ${ }^{6}$ To decrease polymerization shrinkage, the cavities were replaced with resin composite restorations in two steps, as most practitioners advocated. ${ }^{5}$ Aging restorations at body temperature and exposing them to thermocycling and/or mechanical loading are treatment methods commonly used before in vitro microleakage testing to simulate the intraoral service life of a restoration. Different regimens have been used for thermocycling dental restorations with recommended temperatures ranging between $4^{\circ}$ and $60^{\circ}$ C. In the current study all specimens were subjected to 1000 cycles between $5^{\circ} \mathrm{C}$ and $55^{\circ} \mathrm{C}$ with a dwell time of 30 seconds which is considered an appropriate artificial aging test because it is equivalent to 12 months of clinical service. ${ }^{21}$ Several methods have been used to assess the degree of microleakage and the reliability of restorations alongside the margins, such as air pressure, bacterial activity, scanning electron microscope, dye penetration, radioactive isotopes, neutron activation analysis, and microcomputed tomography, can be used to assess microleakage, and each has its own set of benefits and limitations. Some of the previous methods are no longer employed because they cannot accurately model the true nature of microleakage. ${ }^{22}$ The dye penetration method is the most frequently used method for detecting microleakage. ${ }^{4}$ It was chosen for this study because it offers numerous advantages compared with other techniques. First, no radiation or reactive chemicals are used. Second, a variety of dye solutions are accessible making the technique highly feasible and repeatable. Because some dyes, such as basic fuchsin can react with dentin, current research has failed to clearly identify which dyes are appropriate for use with microleakage tests. Another concern with dye penetration methods is the particle size of the dye used which may affect the test reliability. ${ }^{22}$ Methylene blue was chosen because it penetrates further and has a smaller molecule size $(0.5-0.7 \mathrm{~mm}) .{ }^{19}$ However, some authors did not prefer using it as it may lead to an overestimation of the leakage. $^{23}$ While reviewing the findings of this study, we found that all the resin composite materials examined showed dye penetration at the tooth-restoration interface with no statistically significant difference in microleakage between materials. This finding could be due to polymerization shrinkage and changes in the coefficients of thermal expansion and contraction between the tooth and the restorative material. ${ }^{10}$ Internal tensions in the material, as well as leaks between the filler and the cavity walls, are caused by polymerization shrinkage. The elastic modulus of the material in the cavity has been shown to be more important than shrinkage in determining stress specifically, the greater the elasticity of the material in the cavity, the lower the polymerization shrinkage stresses, which may explain the lack of a significant difference in microleakage between the various resin composite materials. $^{24}$ The shape of the cavity (C-factor), orientation of dentinal tubules toward the cervical wall (cementoenamel junction), organic content beneath the dentin layer and movement of dentinal fluid in dentinal tubules, level of hydration of underlying dentin layers, incomplete solvent evaporation from the dentin surface prior to adhesion of adhesive monomers and the composition of acid ( $\mathrm{pH}$, osmolarity, thickening agent), may result in polymerization shrinkage of the composite resins. ${ }^{25}$ Another hypothesis is that the materials share similar properties and consistency. ${ }^{26}$ Nanohybrid resin composites (Tetric N Ceram, Mosaic and Harmonize) showed the least microleakage. These results were consistent with those of Shah et al, who compared nanohybrid resin composites to microfilled resin composites. ${ }^{19}$ According to Shetty et al, nanohybrid resins may provide desired results when compared to other resin composites. This finding is attributable to filler technology, which aids in the reduction of microleakage by contributing to higher strength and fewer spaces between adjacent particles, automatically conferring reduced marginal leakage of bacteria and contaminants. ${ }^{21}$ Omnichroma resin composite showed the highest microleakage in the cervical area and the cavity floor which may depend on the rheological characteristics of the resin composites, monomer types, ratio of the resin matrix and inorganic filler (type and content). ${ }^{18}$ The resin composite materials used in this study showed differences in the resin matrix composition as well as in the fillers, which may influence the properties of the materials, including polymerization shrinkage which has a great impact on microleakage. ${ }^{27}$ Omnichroma has a $79 \mathrm{wt} \%$ filler load which is less than the filler load in the nanohybrid resin composites (Tetric N Ceram has an 80-81 wt\% filler load and Harmonize has an $81 \mathrm{wt} \%$ filler load) Table 1 . The predominant resin matrix in Omnichroma contains UDMA and TEGDMA. The majority of nanohybrid resin composites 
modified their resin composition, such as Harmonize which has (Bis-GMA, Bis-EMA and TEGDMA) or substituted TEGDMA with Bis -GMA (Mosaic) or with UDMA (Tetric N-Ceram) because Bis-GMA and UDMA are high molecular weight particles that considerably reduce shrinkage. ${ }^{21}$ Despite advances in composite resin manufacturing, clinicians face challenges in preventing microleakage due to the variety of tissues involved, operator experience, variability of techniques, and above all, the multiple available protocols. All these factors are important in microleakage assessment according to Falconí-Borja et al. ${ }^{28}$ The findings of this investigation predicted the efficacy of resin composite restorations in vitro, but they did not account for all the potential oral environment variables in vivo. The results of an in vitro microleakage investigation should be regarded as a theoretical maximum level of leakage, which may be higher than what is expected in vivo. As a result, future research could help us understand more about the clinical performance, durability, and efficacy of resin composite restorations. ${ }^{26}$

\section{Conclusions}

Within the limitations of this in vitro study, nanohybrid resin composites showed less microleakage than other resin composites. Tetric N-Ceram showed the least microleakage in the cervical area and Harmonize showed the least microleakage in the cavity floor. Among all the groups, Omnichroma showed the highest microleakage at the cavity floor and cervical areas.

\section{Abbreviations}

Bis-EMA, Bisphenol A Ethoxylate Dimethacrylate; BisGMA, bisphenol A glycidyl methacrylate; nm, nanometer; TEGDMA, triethyleneglycol dimethacrylate; UDMA, urethane dimethacrylate; $\mathrm{SiO} 2-\mathrm{ZrO} 2$, silicon dioxide zirconium dioxide; $\mathrm{Vol} \%$, volume percentage; $\mathrm{Wt} \%$, weight percentage.

\section{Data Sharing Statement}

The data sets used and/or analyzed during the current study are available from the corresponding author on reasonable request.

\section{Ethics Approval and Consent to Participate}

The study has been approved by the ethics committee of Vision College's (approval number: 20-03/8). An informed consent of the patient or the patient's authorized representative prior to extracting procedure was taken in the Oral surgery department- Vision Colleges.

\section{Author Contributions}

All authors made a significant contribution to the work reported, whether that is in the conception, study design, execution, acquisition of data, analysis and interpretation, or in all these areas; took part in drafting, revising or critically reviewing the article; gave final approval of the version to be published; have agreed on the journal to which the article has been submitted; and agree to be accountable for all aspects of the work.

\section{Funding}

Self-funded. This research did not receive any financial support.

\section{Disclosure}

The authors report no conflicts of interest for this work.

\section{References}

1. Dionysopoulos D, Gerasimidou O. Wear of contemporary dental composite resin restorations: a literature review. Restor Dent Endod. 2021;46(2):e18. doi:10.5395/rde.2021.46.e18

2. Hussain S, Raza Khan F. In-vitro comparison of micro-leakage between nanocomposite and micro hybrid composite in class v cavities treated with the self-etch technique. J Ayub Med Coll Abbot. 2016;28(3):445-448.

3. Nematollahi H, Bagherian A, Ghazvini K, Esmaily H, Azadegan M. Microbial microleakage assessment of class $\mathrm{V}$ cavities restored with different materials and techniques: a Laboratory Study. Dent Res J. 2017;14(5):344-350. doi:10.4103/1735-3327.215958

4. Jawaed NU, Abidi SY, Qazi FU, Ahmed S. An in-vitro evaluation of microleakage at the cervical margin between two different class II restorative techniques using dye penetration method. $J$ Coll Physicians Surg Pak. 2016;26(9):748-752. PMID: 27671178.

5. Swapna M, Koshy S, Kumar A, Nanjappa N, Benjamin S, Nainan M. Comparing marginal microleakage of three bulk fill composites in class II cavities using confocal microscope: an in vitro study. J Conserv Dent. 2015;18(5):409-415. doi:10.4103/0972-0707.164058

6. Rengo C, Goracci C, Ametrano G, Chieffi E, Spagnuollo G, Ferrari M. Marginal leakage of class $\mathrm{V}$ composite restorations assessed using microcomputed tomography and scanning electron microscope. Oper Dent. 2015;40(4):440-448. doi:10.2341/14-022-L

7. Ameri H, Chasteen J, Ghavamnasiri M, Maghami A. Effect of load cycling on nanoleakage of butt joint and bevelled occlusal enamel margins in class V composite resin restorations. Rev Clin Odontol. 2010;6(3):231-237. doi:10.7213/aor.v6i3.23160

8. Emir F, Ayyildiz S, Sahin C. What is the changing frequency of diamond burs? J Adv Prosthodont. 2018;10(2):93-100. doi:10.4047/ jap.2018.10.2.93

9. Gupta A, Tavane P, Gupta P, et al. Evaluation of microleakage with total etch, self-etch and universal adhesive systems in class V restorations: an in vitro study. Journal of clinical and diagnostic research. JCDR. 2017;11(4):ZC53. doi:10.7860/JCDR/2017/ 24907.9680 
10. Shetty C, Khanna N, Mithra N. To evaluate and compare the microleakage in class five cavities restored by a nanohybrid and a nanofilled composite resin by dye penetration method-an in vitro study. Indian $J$ Public Health Res Dev. 2019;10 (9):504-508. doi:10.5958/0976-5506.2019.02479.3

11. Phanombualert J, Chimtim P, Heebthamai T, Weera-Archakul W. Microleakage of composite resin restorations in class $\mathrm{V}$ cavities etched by Er: yAGLaser with different pulse modes. Photomed Laser Surg. 2015;33(9):467-472. doi:10.1089/pho.2015.3926

12. Naves L, Gerdolle A, de Andrade S, Gresnigt M. Seeing is believing? When scanning electron microscopy (SEM) meets clinical dentistry: the replica technique. Micro Res Tech. 2020;83(9):1118-1123. doi:10.1002/jemt.23503

13. Magni E, Zhang L, Hickel R, Bossù M, Polimeni A, Ferrari M. SEM and microleakage evaluation of the marginal integrity of two types of class $\mathrm{V}$ restorations with or without the use of a light-curable coating material and of polishing. J Dent. 2008;36(11):885-891. doi:10.1016/ j.jdent.2008.07.003

14. Sheintz S. Clinical relevance of tests on bond strength, microleakage and marginal adaptation. Dent Mat J. 2013;29(1):59-84. doi:10.1016/ j.dental.2012.07.158

15. Alavi A, Kianimanesh N. Microleakage of direct and indirect composite restorations with three dentin bonding agents. Oper Dent. 2002;27(1):19-24. PMID: 11817466.

16. Jacker-Guhr S, Ibarra G, Oppermann L, Lührs A, Rahman A, Geurtsen W. Evaluation of microleakage in Class V composite restorations using dye penetration and micro-CT. Clin Oral Investig. 2016;20(7):1709-1718. doi:10.1007/s00784-015-1676-0

17. Dalli M, Bahsi E, Sahbaz C, et al. A comparison of microleakage scores of five types of composite resins. Biotechnol Biotechnol Equip. 2014;24:2022-2026. doi:10.2478/V10133-010-0072-9

18. Sivakumar J, Prasad A, Soundappan S, Ragavendran N, Ajay R, Santham K. A comparative evaluation of microleakage of restorations using silorane-based dental composite and methacrylate-based dental composites in class II cavities: an in vitro study. J Pharm Bioallied Sci. 2016;8:81-85. doi:10.4103/0975-7406.191975

19. Shah K, Mankar N, Bajaj P, Nikhade P, Chandak M, Gilani R. Comparative evaluation of microleakage in cavities restored with nanohybrid and microfilled composites using oblique incremental technique- an in vitro- study. J Evol Med Dent. 2020;13 (9):1087-1090. doi:10.14260/jemds/2020/234
20. Hegde M, Vyapaka P, Shetty S. A comparative evaluation of microleakage of three different newer direct composite resins using a self-etching primer in class V cavities: an in vitro study. $J$ Conserv Dent. 2009;12(4):160-163. doi:10.4103/0972-0707.58340

21. Wahab F, Shaini F, Morgano S. The effect of thermocycling on microleakage of several commercially available composite class V restorations in vitro. J Prosthet Dent. 2003;90(2):168-174. doi:10.1016/s0022-3913(03)00300-7

22. Alhabdan A. Review of microleakage evaluation tools. J Int Oral Health. 2017;4(9):141-145. doi:10.4103/jioh.jioh_160_17

23. Scotti N, Comba A, Gambino A. Microleakage at enamel and dentin margins with a bulk fills flowable resin. Euro J Dent. 2014;8(1):1-8. doi:10.4103/1305-7456.126230

24. Dwiandhono I, Logamarta S, Hanurdara T. The difference in microleakage levels of nanohybrid composite resin using eighth-generation ethanol and isopropanol solvent bonding materials under moist and dry conditions (in vitro study). Dent J Maj Kedo Gigi. 2019;52 (1):41-44. doi:10.20473/j.djmkg.v52.i1.p41-44

25. Valizadeh S, Hashemi SF, Hashemikamangar S, Kharazifard M. Microleakage of a self-adhesive composite of class V cavities: effect of surface treatment and thermocycling. J Cont Dent Pract. 2020;21 (7):781-786. doi:10.5005/jp-journals-10024-2878

26. Eltoum N, Bakry S, Talaat D, Elshabrawy S. Microleakage evaluation of bulk-fill composite in class II restorations of primary molars. Alex Dent J. 2019;1(44):111-116. doi:10.21608/ ADJALEXU.2019.57614

27. Yazici R, Celik C, Ozgünaltay G. Microleakage of different resin composite types. Quintessence Int. 2004;35(10):790-794.

28. Falconí-Borja G, Molina-Pule C, Velásquez-Ron B, Armas-Vega A. Evaluation of microleakage degree in composite resin restorations by comparing two adhesives systems after different aging periods. Rev Fac Odontol Univ Antioq. 2016;27:281-295.
Clinical, Cosmetic and Investigational Dentistry

\section{Publish your work in this journal}

Clinical, Cosmetic and Investigational Dentistry is an international, peer-reviewed, open access, online journal focusing on the latest clinical and experimental research in dentistry with specific emphasis on cosmetic interventions. Innovative developments in dental materials, techniques and devices that improve outcomes and patient satisfaction and preference will be highlighted. The manuscript management system is completely online and includes a very quick and fair peer-review system, which is all easy to use. Visit

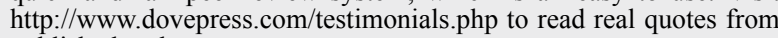
published authors. 